\title{
Absolute Stability of a Class of Nonlinear Singular Systems with Time Delay
}

\author{
Hong-Bing Zeng, Gang Chen, and Shen-Ping Xiao \\ School of Electrical and Information Engineering, Hunan University of Technology, Zhuzhou 412007, China \\ Correspondence should be addressed to Hong-Bing Zeng; 9804zhb@163.com
}

Received 25 December 2013; Accepted 11 March 2014; Published 2 April 2014

Academic Editor: Hao Shen

Copyright ( 2014 Hong-Bing Zeng et al. This is an open access article distributed under the Creative Commons Attribution License, which permits unrestricted use, distribution, and reproduction in any medium, provided the original work is properly cited.

This paper deals with the absolute stability for a class of nonlinear singular systems with time delay. By employing a new LyapunovKrasovskii functional with the idea of partitioning delay length, improved delay-dependent stability criteria are established. The resulting condition is formulated in terms of linear matrix inequalities (LMIs), which is easy to be verified by exiting LMI optimization algorithms. A numerical example is given to show the effectiveness of the proposed technique and its improvements over the existing results.

\section{Introduction}

Since the concept of absolute stability and the Lur'e problem were introduced, the absolute stability of Lur'e control systems has received considerable attention and many rich results have been proposed during the last decades [1]. Time delays widely exist in practical systems, which is a source of instability and deteriorated performance [2-4]. Therefore, great efforts have been made to investigate the absolute stability of Lure systems with time delay and many results have been achieved [4-9].

Recently, an integral inequality approach was proposed to investigate the Lur'e system with time delay and new absolute stability criteria were obtained [7]. In addition, as it is impossible to reduce the conservatism of the derived conditions by employing simple Lyapunov-Krasovskii functional, some other efforts are made to improve the delay-dependent conditions via introducing new Lyapunov-Krasovskii functionals. For example, improved results for time delay systems were obtained by introducing the augmented LyapunovKrasovskii functional [10] and the delay-partitioning Lyapunov-Krasovskii functional [5]. By employing a discretized Lyapunov-Krasovskii functional, new absolute stability condition for a class of nonlinear neutral systems is derived in [11]. Although [11] can achieve less conservative results, the condition was much more complicated than those based on simple Lyapunov-Krasovskii functionals.

On the other hand, singular systems have been extensively studied in the past few years due to the fact that singular systems describe physical systems better than statespace ones [12-15]. Depending on the area of application, these models are also called descriptor systems, semistate systems, differential-algebraic systems, or generalized statespace systems. Therefore, the study of the absolute stability problem for the Lure singular system with time delay is of theoretical and practical importance [16].

In this paper, by employing the delay-partitioning approach proposed in [17], we construct a new LyapunovKrasovskii functional to investigate the absolute stability of Lur'e singular systems with time delay. Improved delaydependent absolute stability criteria are presented. The criteria are easy to follow, and those criteria obtained in [16] by using simple Lyapunov-Krasovskii functional are involved in our results. Numerical example is given to demonstrate the advantage of the proposed method.

Notation. Throughout this paper, $\mathbb{R}^{n}$ denotes the $n$ dimensional Euclidean space; $\mathbb{R}^{n \times m}$ is the set of all $n \times m$ real matrices; for a real matrix $P, P>0$ (resp., $P<0$ ) means that $P$ is real symmetric and positive definite (resp., negative definite); $I$ is an identity matrix of appropriate dimensions, 
and the symmetric terms in a symmetric matrix are denoted by “*."

\section{Problem Statement and Preliminaries}

Consider the following system with time delay and sectorbounded nonlinearity:

$$
\begin{aligned}
E \dot{x}(t) & =A x(t)+B x(t-h)+D w(t), \\
z(t) & =M x(t)+N x(t-h) \\
w(t) & =-\varphi(t, z(t)) \\
x(t) & =\phi(t), \quad t \in[-h, 0]
\end{aligned}
$$

where $x(t) \in \mathbb{R}^{n}$ is the state vector of the system; $w(t) \in$ $\mathbb{R}^{m}$ and $z(t) \in \mathbb{R}^{m}$ are input vector and output vector, respectively; $E, A, B, D, M, N$ are constant matrices, where $E$ may be singular and it is assumed that $\operatorname{rank} E=r \leq n$ and that the scalar $h>0$ is the delay of the system; the initial condition, $\phi(t)$, is a continuous vector-valued function of $t \in[-h, 0]$. $\varphi(t, z(t)) \in \mathbb{R}^{m}$ is a nonlinear function, which is piecewise continuous in $t$, globally Lipschitz in $z(t), \varphi(t, 0)=0$, and satisfies the following sector condition:

$$
\left[\varphi(t, z(t))-K_{1} z(t)\right]^{T}\left[\varphi(t, z(t))-K_{2} z(t)\right] \leq 0,
$$

where $K_{1}$ and $K_{2}$ are constant real matrices and $K=K_{2}-K_{1}$ is a symmetric positive definite matrix. It is customary that such a nonlinear function $\varphi(t, z(t))$ is said to belong to a sector $\left[K_{1}, K_{2}\right]$.

In this paper, we also investigate the robust absolute stability of the following uncertain system:

$$
\begin{aligned}
& E \dot{x}(t)=(A+\Delta A(t)) x(t)+(B+\Delta B(t)) \\
& \times x(t-h)+D w(t), \\
& z(t)= M x(t)+N x(t-h), \\
& w(t)=-\varphi(t, z(t)), \\
& x(t)=\phi(t), \quad t \in[-h, 0],
\end{aligned}
$$

where the uncertainties are of the form

$$
[\Delta A(t) \Delta B(t)]=L F(t)\left[\begin{array}{ll}
E_{a} & E_{b}
\end{array}\right]
$$

where $L, E_{a}$, and $E_{b}$ are constant matrices, and $F(t)$ is a timevarying matrix satisfying

$$
F^{T}(t) F(t) \leq I, \quad \forall t
$$

Next, the following definitions and lemmas are introduced, which will be used in the proof of the main results.

Definition 1 (see [12]). (i) The pair $(E, A)$ is said to be regular if $\operatorname{det}(s E-A)$ is not identically zero. (ii) The pair $(E, A)$ is said to be impulse-free if $\operatorname{deg}(\operatorname{det}(s E-A))=\operatorname{rank} E$.

Definition 2 (see [12]). (i) The nonlinear singular system (1) is said to be regular and impulse-free if the pair $(E, A)$ is regular and impulse-free. (ii) The nonlinear singular system (1) is said to be globally uniformly asymptotically stable for any nonlinear function $\varphi(t, z(t))$ satisfying (2) if, for any $\epsilon>$ 0 , there exists a scalar $\delta(\epsilon)$ such that, for any compatible initial conditions $\phi(t)$ satisfying $\sup _{-h \leq t \leq 0}\|\phi(t)\| \leq \delta(\epsilon)$, the solution $x(t)$ of the system (1) satisfies $\|x(t)\| \leq \epsilon$ for $t \geq 0$. Furthermore, $\lim _{t \rightarrow \infty} x(t)=0$.

Lemma 3 (see [18]). Consider the function $\varphi: \mathbb{R}^{+} \rightarrow \mathbb{R} ;$ if $\varphi$ is uniformly continuous and $\int_{0}^{\infty} \varphi(s) d s<\infty, \lim _{t \rightarrow \infty} \varphi(t)=$ 0 .

Lemma 4 (see [19]). For any symmetric positive-definite matrix $M \in \mathbb{R}^{n \times n}$ and a scalar $\gamma>0$, if there exists a vector function $\omega(\alpha):[-\gamma, 0] \rightarrow \mathbb{R}^{n}$ such that the following integrals are well defined, then

$$
\begin{aligned}
& -\gamma \int_{-\gamma}^{0} \dot{\omega}(t+\alpha)^{T} E^{T} M E \dot{\omega}(t+\alpha) d \alpha \\
& \quad \leq\left[\begin{array}{c}
\omega(t) \\
\omega(t-\gamma)
\end{array}\right]^{T}\left[\begin{array}{cc}
-E^{T} M E & E^{T} M E \\
* & -E^{T} M E
\end{array}\right]\left[\begin{array}{c}
\omega(t) \\
\omega(t-\gamma)
\end{array}\right] .
\end{aligned}
$$

Lemma 5 (see [20]). Let $H, E$, and $F(t)$ be real matrices of appropriate dimensions with $F(t)$ satisfying $F^{T}(t) F(t) \leq I$. Then, for any scalar $\varepsilon>0$,

$$
H F(t) E+(H F(t) E)^{T} \leq \varepsilon^{-1} H H^{T}+\varepsilon E^{T} E .
$$

\section{Main Results}

Firstly, by means of the loop transformation suggested in [21], it can be concluded that the absolute stability of system (1) in the sector $\left[K_{1}, K_{2}\right]$ is equivalent to that of the following system in the sector $\left[0, K_{2}-K_{1}\right]$ :

$$
\begin{aligned}
E \dot{x}(t) & =\bar{A} x(t)+\bar{B} x(t-h)+D w(t), \\
z(t) & =M x(t)+N x(t-h), \\
w(t) & =-\varphi(t, z(t)) \\
x(t) & =\phi(t), \quad t \in[-h, 0]
\end{aligned}
$$

where $\bar{A}=A-D K_{1} M, \bar{B}=B-D K_{1} N$.

Thus, for the absolute stability of system (1), we have the following result.

Theorem 6. Given integer $k$ and scalar $\tau=h / k>0$, the system (1) with nonlinear connection function satisfying (2) is absolutely stable in the sector $\left[K_{1}, K_{2}\right]$ if there exist a scalar $\varepsilon>0$, matrices

$$
\begin{gathered}
P=P^{T}>0 \\
Q_{a}=\left[\begin{array}{cccc}
Q_{11} & Q_{12} & \cdots & Q_{1 k} \\
* & Q_{22} & \cdots & Q_{2 k} \\
* & * & \ddots & \vdots \\
* & * & * & Q_{k k}
\end{array}\right] \geq 0, \\
Z_{i}=Z_{i}^{T}>0, \quad(i=1,2 \ldots, k),
\end{gathered}
$$


and a matrix $S$ with appropriate dimensions, such that the following LMI holds:

$$
\Phi=\left[\begin{array}{ccc}
\Phi_{11} & \Phi_{12} & \Phi_{13} \\
* & \Phi_{22} & 0 \\
* & * & \Phi_{33}
\end{array}\right]<0,
$$

where

$$
\begin{aligned}
& \Phi_{11}=\left[\begin{array}{ccc}
\Pi_{1} & \widetilde{P} \bar{B}+E^{T} Z_{1} E & \widetilde{P} D-\varepsilon M^{T}\left(K_{2}-K_{1}\right)^{T} \\
* & -Q_{k k}-E^{T} Z_{k} E & -\varepsilon N^{T}\left(K_{2}-K_{1}\right)^{T} \\
* & * & -2 \varepsilon I
\end{array}\right], \\
& \Phi_{12}=\left[\begin{array}{cccc}
E^{T} Z_{1} E+Q_{12} & Q_{13} & \ldots & Q_{1 k} \\
-Q_{1 k}^{T} & \cdots & -Q_{(k-2) k}^{T} & E^{T} Z_{k} E-Q_{(k-1) k}^{T} \\
0 & \cdots & 0 & 0
\end{array}\right] \text {, } \\
& \Pi_{1}=\widetilde{P} \bar{A}+\bar{A}^{T} \widetilde{P}+Q_{11}-E^{T} Z_{1} E, \quad \widetilde{P}=E^{T} P+S R^{T}, \\
& \Phi_{22}=\left[\begin{array}{ccccc}
\Lambda_{1} & \bar{\Lambda}_{1} & \bar{Q}_{13} & \cdots & \bar{Q}_{1(k-1)} \\
* & \Lambda_{2} & \bar{\Lambda}_{2} & \ldots & \vdots \\
* & * & \ddots & \ddots & \bar{Q}_{(k-3)(k-1)} \\
* & * & * & \Lambda_{k-2} & \bar{\Lambda}_{k-2} \\
* & * & * & * & \Lambda_{k-1}
\end{array}\right], \\
& \bar{Q}_{i j}=Q_{(i+1)(j+1)}-Q_{i j}, \quad \Lambda_{i}=-E^{T} Z_{i} E-E^{T} Z_{i+1} E+\bar{Q}_{i i} \text {, } \\
& (i, j=1,2, \ldots, k-1), \\
& \bar{\Lambda}_{i}=E^{T} Z_{i+1} E+\bar{Q}_{i(i+1)}, \quad(i=1,2, \ldots, k-2), \\
& \Phi_{13}=\tau \Gamma^{T} \sum_{i=1}^{k} Z_{i}, \quad \Phi_{33}=-\sum_{i=1}^{k} Z_{i}, \quad \Gamma=\left[\begin{array}{lll}
\bar{A} & \bar{B} & D
\end{array}\right]
\end{aligned}
$$

and $R \in \mathbb{R}^{n \times(n-r)}$ is any matrix with full column rank and satisfying $R^{T} E=0$.

Proof. Firstly, dividing the delay $h$ into $k$ equal segments, the length of each segment is denoted as $\tau$; that is, $\tau=h / k$. Choosing a Lyapunov-Krasovskii functional is as follows:

$$
\begin{aligned}
V\left(t, x_{t}\right)= & x^{T}(t) E^{T} \operatorname{PEx}(t)+\int_{t-\tau}^{t} \zeta_{1}^{T}(s) Q_{a} \zeta_{1}(s) d s \\
& +\sum_{i=1}^{k} \int_{-i \tau}^{-(i-1) \tau} \int_{t+\theta}^{t} \tau \dot{x}^{T}(s) E^{T} Z_{i} E \dot{x}(s) d s d \theta,
\end{aligned}
$$

where

$$
\begin{gathered}
P>0 \\
Q_{a}=\left[\begin{array}{cccc}
Q_{11} & Q_{12} & \cdots & Q_{1 k} \\
* & Q_{22} & \cdots & Q_{2 k} \\
* & * & \ddots & \vdots \\
* & * & * & Q_{k k}
\end{array}\right] \geq 0, \\
Z_{i}>0, \quad(i=1,2, \ldots, k)
\end{gathered}
$$

are to be determined and $\zeta_{1}(t)=\left[\begin{array}{lll}x^{T}(t) & x^{T}(t-\tau) & \cdots\end{array}\right.$ $\left.x^{T}(t-(k-1) \tau)\right]^{T}$.

Calculating the derivative of each $V\left(t, x_{t}\right)$ along the solutions of system (8) yields

$$
\begin{aligned}
\dot{V}\left(t, x_{t}\right)= & x^{T}(t)\left(E^{T} P \bar{A}+\bar{A}^{T} P E\right) x(t) \\
& +2 x^{T}(t) E^{T} P \bar{B} x(t-h)+2 x^{T}(t) E^{T} P D w(t) \\
& +\zeta_{1}^{T}(t) Q_{a} \zeta_{1}(t)-\zeta_{1}^{T}(t-\tau) Q_{a} \zeta_{1}(t-\tau) \\
& +\tau^{2} \dot{x}^{T}(t) \sum_{i=1}^{k} E^{T} Z_{i} E \dot{x}(t) \\
& -\sum_{i=1}^{k} \int_{t-i \tau}^{t-(i-1) \tau} \tau \dot{x}^{T}(s) E^{T} Z_{i} E \dot{x}(s) d s .
\end{aligned}
$$

Let $\theta=-\int_{t-i \tau}^{t-(i-1) \tau} \tau \dot{x}^{T}(s) E^{T} Z_{i} E \dot{x}(s) d s$; using Lemma 4, we have

$$
\begin{aligned}
\theta \leq & {\left[\begin{array}{c}
x(t-(i-1) \tau) \\
x(t-i \tau)
\end{array}\right]^{T}\left[\begin{array}{cc}
-E^{T} Z_{i} E & E^{T} Z_{i} E \\
* & -E^{T} Z_{i} E
\end{array}\right] } \\
& \times\left[\begin{array}{c}
x(t-(i-1)) \tau \\
x(t-i \tau)
\end{array}\right] .
\end{aligned}
$$

From (1) and (2), for $\varphi(t, z(t)) \in\left[0, K_{2}-K_{1}\right]$ and a scalar $\varepsilon>0$, it can be deduced that

$$
\begin{aligned}
0 \leq & -2 \varepsilon w^{T}(t) w(t)-2 \varepsilon w^{T}(t)\left(K_{2}-K_{1}\right) \\
& \times[M x(t)+N x(t-h)] .
\end{aligned}
$$

Noting that $R^{T} E=0$, we can deduce

$$
0=R^{T} \bar{A} x(t)+R^{T} \bar{B} x(t-h)+R^{T} D w(t) .
$$

From (14)-(17), we get

$$
\dot{V}\left(t, x_{t}\right) \leq \zeta^{T}(t)\left[\Psi+\tau^{2} \bar{\Gamma}^{T} \sum_{i=1}^{k} Z_{i} \bar{\Gamma}\right] \zeta(t),
$$

where

$$
\begin{gathered}
\Psi=\left[\begin{array}{cc}
\Phi_{11} & \Phi_{12} \\
* & \Phi_{22}
\end{array}\right], \quad \bar{\Gamma}=\left[\begin{array}{llll}
\bar{A} & \bar{B} & D & 0
\end{array}\right] \\
\zeta(t)=\left[\begin{array}{llll}
x^{T}(t) & x^{T}(t-h) & w^{T}(t) & \zeta_{2}^{T}(t)
\end{array}\right]^{T}, \\
\zeta_{2}^{T}(t)=\left[\begin{array}{llll}
x^{T}(t-\tau) & x^{T}(t-2 \tau) & \cdots & x^{T}(t-(k-1) \tau)
\end{array}\right]^{T} .
\end{gathered}
$$

If $\Psi+\tau^{2} \bar{\Gamma}^{T} \sum_{i=1}^{k} Z_{i} \bar{\Gamma}<0$, which is equivalent to (10) by Schur complements [22], then $\dot{V}\left(t, x_{t}\right)<0$ holds.

In what follows, we show that the nonlinear singular system (1) is regular and impulse-free. Since rank $E=r \leq n$, there exist two invertible matrices $G$ and $H \in \mathbb{R}^{n \times n}$ such that

$$
\bar{E}=G E H=\left[\begin{array}{cc}
I_{r} & 0 \\
0 & 0
\end{array}\right] .
$$


Then, $R$ can be parameterized as

$$
R=G^{T}\left[\begin{array}{c}
0 \\
\bar{\Phi}
\end{array}\right]
$$

where $\bar{\Phi} \in \mathbb{R}^{(n-r) \times(n-r)}$ is any nonsingular matrix.

Like in (20), we define

$$
\begin{aligned}
& \bar{A}=G A H=\left[\begin{array}{ll}
\bar{A}_{11} & \bar{A}_{12} \\
\bar{A}_{21} & \bar{A}_{22}
\end{array}\right], \\
& \bar{P}=G^{-T} P G^{-1}=\left[\begin{array}{ll}
\bar{P}_{11} & \bar{P}_{12} \\
\bar{P}_{21} & \bar{P}_{22}
\end{array}\right], \\
& \bar{Z}_{i}=G^{-T} Z_{i} G^{-1}=\left[\begin{array}{ll}
\bar{Z}_{i 11} & \bar{Z}_{i 12} \\
\bar{Z}_{i 21} & \bar{Z}_{i 22}
\end{array}\right], \quad(i=1,2, \ldots, k), \\
& \bar{S}=H^{T} S=\left[\begin{array}{l}
\bar{S}_{11} \\
\bar{S}_{21}
\end{array}\right], \quad \bar{R}=G^{-T} R=\left[\begin{array}{c}
0 \\
\bar{\Phi}
\end{array}\right] .
\end{aligned}
$$

Since $A^{T}\left(P E+R S^{T}\right)+\left(E^{T} P+S R^{T}\right) A+Q_{11}-E^{T} Z_{1} E<0$ and $Q_{11} \geq 0$, we can formulate the following inequality easily:

$$
\psi=A^{T}\left(P E+R S^{T}\right)+\left(E^{T} P+S R^{T}\right) A-E^{T} Z_{1} E<0
$$

Pre- and postmultiplying $\psi<0$ by $H^{T}$ and $H$, respectively, yield

$$
\begin{aligned}
\bar{\psi} & =H^{T} \psi H=\bar{A}^{T} \bar{P} \bar{E}+\bar{A}^{T} \bar{R} \bar{S}^{T}+\bar{E}^{T} \bar{P} \bar{A}+\bar{S} \bar{R}^{T} \bar{A}-\bar{E}^{T} \bar{Z}_{1} \bar{E} \\
& =\left[\begin{array}{cc}
\bar{\psi}_{11} & \bar{A}_{12}^{T} \bar{\Phi}_{21} \bar{S}_{21}^{T}+\bar{S}_{21} \bar{\Phi}^{T} \bar{A}_{22}
\end{array}\right]<0 .
\end{aligned}
$$

As $\bar{\psi}_{11}$ and $\bar{\psi}_{12}$ are irrelevant to the results of the following discussion, the expressions about these two variables are omitted here. It is easy to deduce from (24) that

$$
\bar{A}_{22}^{T} \bar{\Phi}^{T} \bar{S}_{21}^{T}+\bar{S}_{21} \bar{\Phi}^{T} \bar{A}_{22}<0
$$

and thus $\bar{A}_{22}$ is nonsingular. Otherwise, supposing $A_{22}$ is singular, there must exist a nonzero vector $\varsigma \in \mathbb{R}^{n-r}$ which ensures that $\bar{A}_{22} \varsigma=0$. And then it can be concluded that $\varsigma^{T}\left(\bar{A}_{22}^{T} \bar{\Phi} \bar{S}_{21}^{T}+\bar{S}_{21} \bar{\Phi}^{T} \bar{A}_{22}\right) \varsigma=0$, and this contradicts (25). So $\bar{A}_{22}$ is nonsingular. Then, it can be shown that

$$
\begin{aligned}
\operatorname{det}(s E-A)= & \operatorname{det}\left(G^{-1}\right) \operatorname{det}(s \bar{E}-\bar{A}) \operatorname{det}\left(H^{-1}\right) \\
= & \operatorname{det}\left(G^{-1}\right) \operatorname{det}\left(-\bar{A}_{22}\right) \\
& \times \operatorname{det}\left(s I_{r}-\left(\bar{A}_{11}-\bar{A}_{12} \bar{A}_{22}^{-1} \bar{A}_{21}\right)\right) \operatorname{det}\left(H^{-1}\right)
\end{aligned}
$$

which implies that $\operatorname{det}(s E-A)$ is not identically zero and $\operatorname{deg}(\operatorname{det}(s E-A))=r=\operatorname{rank} E$. Then, the pair of $(E, A)$ is regular and impulse-free, which implies that system (1) is regular and impulse-free.

$$
\begin{aligned}
\text { Defining } \xi(t)=\left[\begin{array}{l}
\xi_{1}(t) \\
\xi_{2}(t)
\end{array}\right] & =H^{-1} x(t) \text {, then we have } \\
\bar{\lambda}_{1}\left\|\xi_{1}(t)\right\|^{2}-V(x(0)) & \leq \xi^{T}(t) \bar{E}^{T} \bar{P} \bar{E} \xi(t)-V(x(0)) \\
& =x^{T}(t) E^{T} P E x(t)-V(x(0)) \\
& \leq V(x(t))-V(x(0)) \\
& =\int_{0}^{t} \dot{V}(x(s)) d s \\
& \leq-\bar{\lambda}_{2} \int_{0}^{t}\|x(s)\|^{2} d s \\
& \leq-\bar{\lambda}_{2}\|H\|^{2} \int_{0}^{t}\|\xi(s)\|^{2} d s
\end{aligned}
$$

where $\bar{\lambda}_{1}=\lambda_{\text {min }}\left(\bar{P}_{11}\right), \bar{\lambda}_{2}=-\lambda_{\max }(\Phi)$.

Taking into account (27), we can deduce that

$$
\bar{\lambda}_{1}\left\|\xi_{1}(t)\right\|^{2}+\bar{\lambda}_{2}\|H\|^{2} \int_{0}^{t}\|\xi(s)\|^{2} d s \leq V(x(0)) .
$$

Noting that $\|x(t)\|$ and $\int_{0}^{t}\|x(s)\|^{2} d s$ are bounded, it follows that $\|\xi(t)\|$ and $\int_{0}^{t}\|\xi(s)\|^{2} d s$ are bounded; from Lemma 3, one can conclude that $\lim _{t \rightarrow \infty} \xi(t)=0$; thus $\lim _{t \rightarrow \infty} x(t)=0$. According to Definition 2, the singular system (8) is globally uniformly asymptotically stable for $\varphi(t, z(t)) \in\left[0, K_{2}-\right.$ $K_{1}$ ]. Thus the singular system (8) is absolutely stable in the sector $\left[0, K_{2}-K_{1}\right]$, which is equivalent to the absolute stability of system (1) in the sector $\left[K_{1}, K_{2}\right]$. This completes the proof.

For uncertain system (3), substituting $A+L F(t) E_{a}$ and $B+L F(t) E_{b}$ for $A$ and $B$ in (10) and utilizing Lemma 5 and Schur complements [22], we have the following result.

Theorem 7. Given integer $k$ and scalar $\tau=h / k>0$, the system (3) with nonlinear connection function satisfying (2) and time-varying structured uncertainties satisfying (4) is robustly absolutely stable in the sector $\left[K_{1}, K_{2}\right]$ if there exist scalars $\varepsilon>0, \lambda>0$, matrices

$$
\begin{gathered}
P=P^{T}>0 \\
Q_{a}=\left[\begin{array}{cccc}
Q_{11} & Q_{12} & \cdots & Q_{1 k} \\
* & Q_{22} & \cdots & Q_{2 k} \\
* & * & \ddots & \vdots \\
* & * & * & Q_{k k}
\end{array}\right] \geq 0, \\
Z_{i}=Z_{i}^{T}>0, \quad(i=1,2 \ldots, k),
\end{gathered}
$$

and a matrix $S$ with appropriate dimensions, such that the following LMI holds:

$$
\left[\begin{array}{ccccc}
\Phi_{11} & \Phi_{12} & \Phi_{13} & \widehat{P} L & \lambda \widehat{E} \\
* & \Phi_{22} & 0 & 0 & 0 \\
* & * & \Phi_{33} & \tau \sum_{i=1}^{k} Z_{i} L & 0 \\
* & * & * & -\lambda I & 0 \\
* & * & * & * & -\lambda I
\end{array}\right]<0,
$$


TABLE 1: Maximum upper bounds of $h$.

\begin{tabular}{|c|c|c|c|c|c|c|}
\hline & \multicolumn{6}{|c|}{$\alpha$} \\
\hline & 0.15 & 0.5 & 1 & 1.5 & 2.5 & 3.5 \\
\hline$[16$, Theorem 3] & 2.6556 & 2.3358 & 1.9489 & 1.6352 & 1.1780 & 0.8749 \\
\hline Theorem $7, k=2$ & 3.7209 & 3.2715 & 2.7269 & 2.2848 & 1.6396 & 1.2103 \\
\hline Theorem $7, k=3$ & 3.9351 & 3.4592 & 2.8824 & 2.4141 & 1.7303 & 1.2748 \\
\hline Theorem $7, k=4$ & 4.0114 & 3.5262 & 2.9379 & 2.4600 & 1.7625 & 1.2976 \\
\hline
\end{tabular}

where

$$
\widehat{P}=\left[\begin{array}{c}
\left(E^{T} P+S R^{T}\right) \\
0 \\
0
\end{array}\right], \quad \widehat{E}=\left[\begin{array}{c}
E_{a}^{T} \\
E_{b}^{T} \\
0
\end{array}\right]
$$

and $R \in \mathbb{R}^{n \times(n-r)}$ is any matrix with full column rank and satisfying $R^{T} E=0 ; \Phi_{11}, \Phi_{12}, \Phi_{13}, \Phi_{22}, \Phi_{33}$ are defined in Theorem 6.

Remark 8. It is worth mentioning that the conservatism is reduced with the increase of $k$. At the same time, more matrix variables are involved in the corresponding LMI, which will increase the computing complexity.

Remark 9. In [5], some absolute stability conditions have been obtained for Lur'e system with time delay based on a delay-partitioning approach. However, the results proposed in this paper achieve some improvement and are more general than [5]. Let $E=I, S=0$, and $Q_{i j}=0(i \neq j)$ in (30); Theorem 7 reduces to Theorem 3 in [5].

\section{Numerical Example}

In this section, we provide a numerical example to demonstrate the effectiveness of the proposed method.

Example 10. Consider uncertain system (3) with the following parameters:

$$
\begin{aligned}
& E=\left[\begin{array}{ll}
1 & 0 \\
0 & 0
\end{array}\right], \quad A=\left[\begin{array}{cc}
0.5 & 0 \\
0 & -1
\end{array}\right], \\
& B=\left[\begin{array}{cc}
-1.1 & 1 \\
0 & 0.5
\end{array}\right], \quad D=\left[\begin{array}{cc}
0.2 & 0 \\
0 & 0.1
\end{array}\right] \text {, } \\
& M=\left[\begin{array}{cc}
0.4 & 0 \\
0 & 0.5
\end{array}\right], \quad N=\left[\begin{array}{cc}
0.2 & 0 \\
0 & 0.1
\end{array}\right] \text {, } \\
& K_{1}=\left[\begin{array}{cc}
0.1 & 0 \\
0 & 0.2
\end{array}\right], \quad K_{2}=\left[\begin{array}{cc}
0.2 & 0 \\
0 & 0.5
\end{array}\right] \text {, } \\
& L=\left[\begin{array}{cc}
\alpha & 0 \\
0 & \alpha
\end{array}\right], \quad \alpha \geq 0, \quad E_{a}=E_{b}=\left[\begin{array}{cc}
0.1 & 0 \\
0 & 0.1
\end{array}\right] \text {. }
\end{aligned}
$$

In this example, we choose $R=\left[\begin{array}{ll}0 & 1\end{array}\right]^{T}$. For various $\alpha$, the maximum upper bounds of time delay obtained by Theorem 7 are listed in Table 1 in comparison with those obtained by [16]. It is clear that our approach provides larger stability region than [16]. Furthermore, it is concluded from the table that larger upper bounds of $h$ can be obtained as $k$ increases.

\section{Conclusions}

The absolute stability problem has been investigated for time delay singular systems with sector-bounded nonlinearity. Some improved conditions have been derived based on the delay-partitioning approach. A numerical example has been given to verify the effectiveness of the proposed methods.

\section{Conflict of Interests}

The authors declare that there is no conflict of interests regarding the publication of this paper.

\section{Acknowledgments}

This work was supported by the National Natural Science Foundation of China (nos. 61304064, 61273157, 61074067, and 61262032) and the Natural Science Foundation of Hunan University of Technology (no. 2012HZX06).

\section{References}

[1] A. I. Lur'e, Some Nonlinear Problems in the Theory of Automatic Control, H.M. Stationery Office, 1957.

[2] H. Shen, S. Xu, X. Song, and J. Luo, "Delay-dependent robust stabilization for uncertain stochastic switching systems with distributed delays," Asian Journal of Control, vol. 11, no. 5, pp. 527-535, 2009.

[3] H. Shen, S. Xu, J. Zhou, and J. Lu, "Fuzzy $H_{\infty}$ filtering for nonlinear Markovian jump neutral systems," International Journal of Systems Science, vol. 42, no. 5, pp. 767-780, 2011.

[4] H.-B. Zeng, Y. He, M. Wu, and S.-P. Xiao, "Absolute stability and stabilization for Lurie networked control systems," International Journal of Robust and Nonlinear Control, vol. 21, no. 14, pp. 16671676, 2011.

[5] M. Wu, Z. Y. Feng, and Y. He, "Improved delay-dependent absolute stability of Lur'e systems with time-delay," International Journal of Control, Automation, and Systems, vol. 7, no. 6, pp. 1009-1014, 2009.

[6] A. Kazemy and M. Farrokhi, "Robust absolute stability analysis of multiple time-delay Lur'e systems with parametric uncertainties," Asian Journal of Control, vol. 15, no. 1, pp. 203-213, 2013.

[7] Q.-L. Han, "Absolute stability of time-delay systems with sectorbounded nonlinearity," Automatica, vol. 41, no. 12, pp. 21712176,2005 . 
[8] Y. He, M. Wu, J.-H. She, and G.-P. Liu, "Robust stability for delay Lur'e control systems with multiple nonlinearities," Journal of Computational and Applied Mathematics, vol. 176, no. 2, pp. 371380, 2005.

[9] Q.-L. Han and D. Yue, "Absolute stability of Lur'e systems with time-varying delay," IET Control Theory \& Applications, vol. 1, no. 3, pp. 854-859, 2007.

[10] Y. He, Q.-G. Wang, C. Lin, and M. Wu, "Augmented Lyapunov functional and delay-dependent stability criteria for neutral systems," International Journal of Robust and Nonlinear Control, vol. 15, no. 18, pp. 923-933, 2005.

[11] Q.-L. Han, "A new delay-dependent absolute stability criterion for a class of nonlinear neutral systems," Automatica, vol. 44, no. 1, pp. 272-277, 2008.

[12] L. Dai, Singular Control Systems, Springer, Berlin, Germany, 1989.

[13] C. Yang, Q. Zhang, and L. Zhou, "Generalised absolute stability analysis and synthesis for Lur'e-type descriptor systems," IET Control Theory \& Applications, vol. 1, no. 3, pp. 617-623, 2007.

[14] S. Xu, P. van Dooren, R. Ştefan, and J. Lam, "Robust stability and stabilization for singular systems with state delay and parameter uncertainty," IEEE Transactions on Automatic Control, vol. 47, no. 7, pp. 1122-1128, 2002.

[15] Z.-G. Wu and W.-N. Zhou, "Delay-dependent robust stabilization for uncertain singular systems with state delay," Acta Automatica Sinica, vol. 33, no. 7, pp. 714-718, 2007.

[16] H. Wang, A. Xue, and R. Lu, "Absolute stability criteria for a class of nonlinear singular systems with time delay," Nonlinear Analysis: Theory, Methods \& Applications, vol. 70, no. 2, pp. 621630, 2009.

[17] F. Gouaisbaut and D. Peaucelle, "Delay-dependent stability analysis of linear time delay systems," in Proceedings of the 6th IFAC Workshop on Time-Delay Systems, 2006.

[18] M. Krstić and H. Deng, Stabilization of Nonlinear Uncertain Systems, Springer, London, UK, 1998.

[19] Z. Wu, H. Su, and J. Chu, " $H_{\infty}$ filtering for singular Markovian jump systems with time delay," International Journal of Robust and Nonlinear Control, vol. 20, no. 8, pp. 939-957, 2010.

[20] I. R. Petersen and C. V. Hollot, "A Riccati equation approach to the stabilization of uncertain linear systems," Automatica, vol. 22, no. 4, pp. 397-411, 1986.

[21] H. K. Khalil, Nonlinear Systems, Prentice Hall, Englewood Cliffs, NJ, USA, 1996.

[22] S. Boyd, L. El Ghaoui, E. Feron, and V. Balakrishnan, Linear Matrix Inequalities in System and Control Theory, SIAM, Philadelphia, Pa, USA, 1994. 


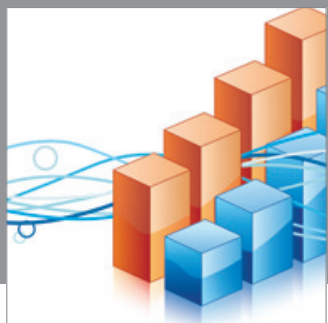

Advances in

Operations Research

mansans

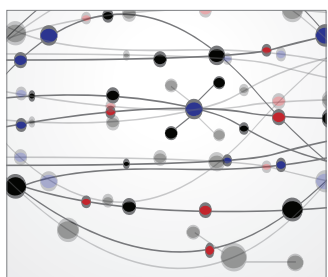

The Scientific World Journal
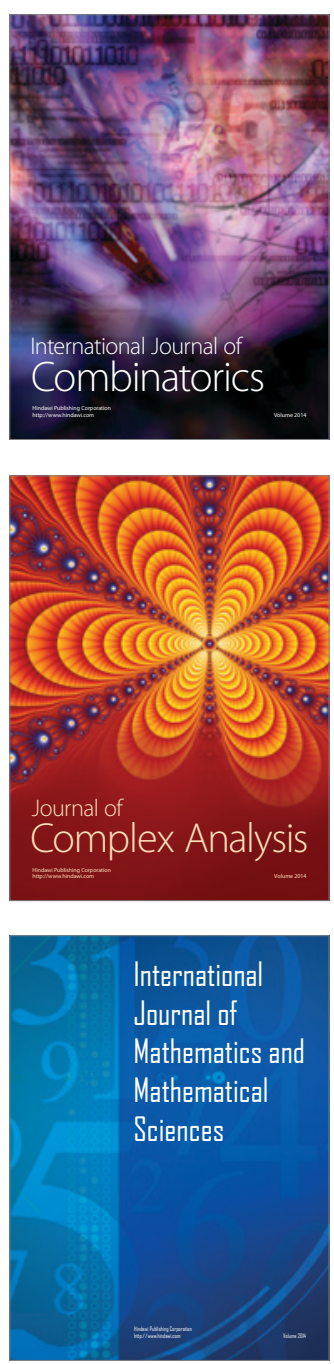
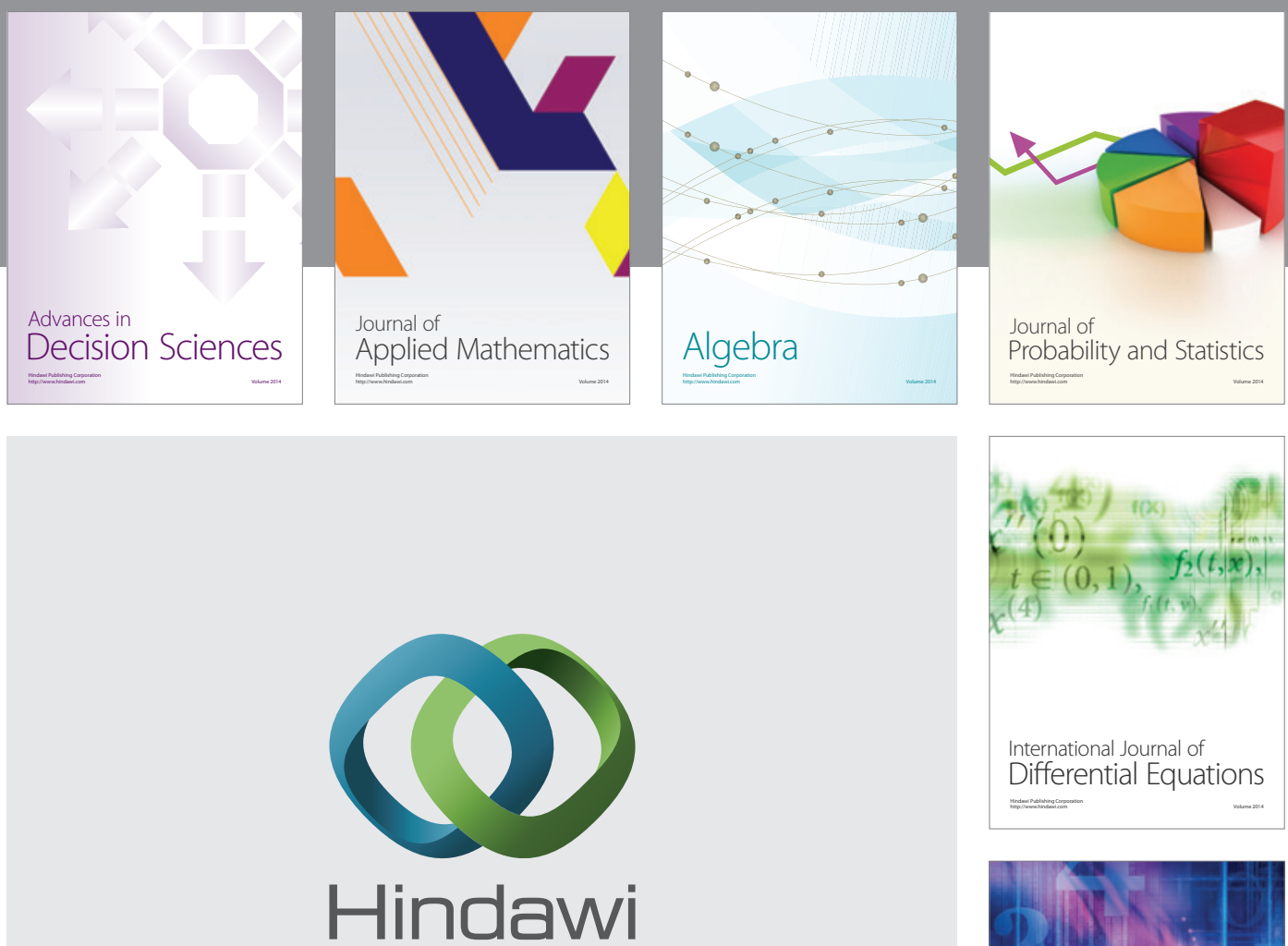

Submit your manuscripts at http://www.hindawi.com
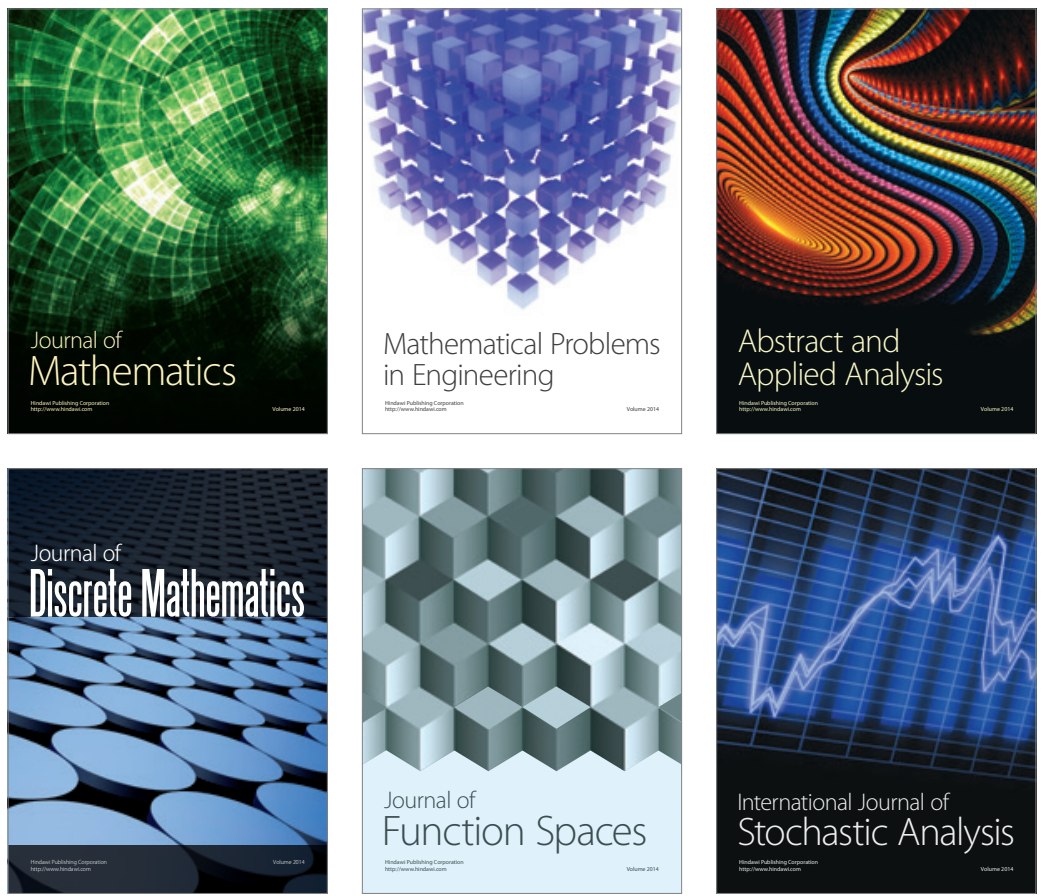

Journal of

Function Spaces

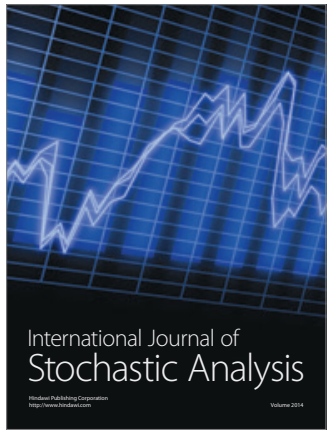

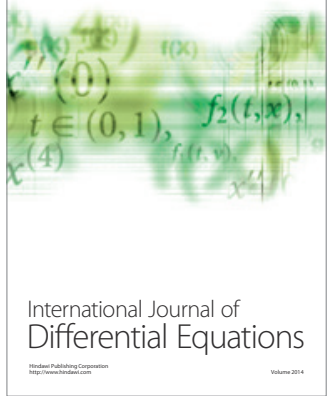
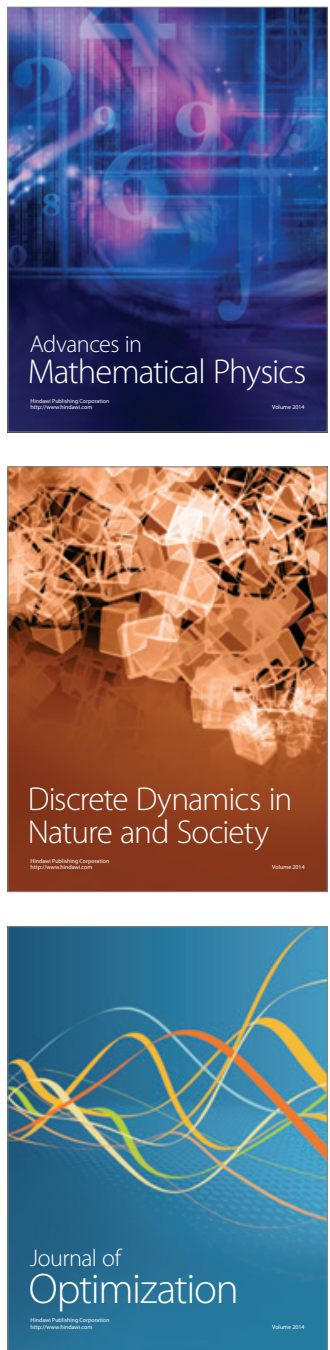